\title{
Impact of the time from the completion of neoadjuvant chemotherapy to surgery on the outcomes of patients with gastric cancer
}

\author{
Chaorui Wu, Hong Zhou, Tongbo Wang, Xiaojie Zhang, Yingtai Chen, Dongbing Zhao \\ Department of Pancreatic and Gastric Surgery, National Cancer Center/National Clinical Research Center for Cancer/Cancer Hospital, Chinese \\ Academy of Medical Sciences and Peking Union Medical College, Beijing 100021, China \\ Contributions: (I) Conception and design: C Wu, D Zhao; (II) Administrative support: D Zhao; (III) Provision of study materials or patients: T Wang, \\ H Zhou, Y Chen; (IV) Collection and assembly of data: T Wang, H Zhou, X Zhang; (V) Data analysis and interpretation: C Wu, D Zhao; Y Chen; (VI) \\ Manuscript writing: All authors; (VII) Final approval of manuscript: All authors. \\ Correspondence to: Yingtai Chen, MD. National Cancer Center/National Clinical Research Center for Cancer/Cancer Hospital, Chinese Academy \\ of Medical Sciences and Peking Union Medical College, Beijing 100021, China. Email: yingtai.chen@hotmail.com; Dongbing Zhao, MD. National \\ Cancer Center/National Clinical Research Center for Cancer/Cancer Hospital, Chinese Academy of Medical Sciences and Peking Union Medical \\ College, Beijing 100021, China. Email: dbzhao@cicams.ac.cn.
}

Background: Gastrectomy is usually recommended within 5 to 6 weeks after the completion of neoadjuvant chemotherapy (NCT). However, the optimal timing of surgery is not clearly defined.

Methods: This study retrospectively reviewed the clinical records of 229 patients with locally advanced gastric cancer (GC) who underwent curative gastrectomy after NCT between 2006 and 2016. The effect of the time to surgery (TTS), defined as $\leq 4,5-6$, and $>6$ weeks, on patient outcomes was examined. Descriptive statistics and Cox proportional hazards models were used.

Results: Seventy of 229 patients (30.6\%) had surgery within 4 weeks after their last dose of NCT, 103 $(45.0 \%)$ within 5-6 weeks, and $56(24.5 \%)$ after 6 weeks. The median age was 56.0 [interquartile range (IQR), 47.0-63.0] years, and the median TTS was 34.0 (IQR, 26.0-42.0) days. The three groups did not significantly differ regarding most surgical and histopathological characteristics except for the NCT regimen $(\mathrm{P}=0.010)$, number of cycles of NCT $(\mathrm{P}=0.017)$ and pathological stage $(\mathrm{P}=0.015)$. The NCT regimen was the only independent factor associated with TTS $>6$ weeks $(\mathrm{P}=0.015)$. The 3 -year progression-free survival (PFS) estimates were $41.9 \%, 42.8 \%$ and $61.7 \%$ in patients who underwent surgery in $\leq 4,5-6$, and $>6$ weeks after NCT, respectively (P=0.044). The 3-year overall survival (OS) estimates were $57.7 \%, 58.0 \%$ and $68.2 \%$ in the three groups, respectively $(\mathrm{P}=0.202)$. According to multivariable analysis, compared with the interval of $\leq 4$ weeks, patients who underwent surgery at 5-6 or $>6$ weeks had equivalent PFS and OS.

Conclusions: The NCT-surgery interval time has no impact on patient outcomes.

Keywords: Gastric cancer (GC); Neoadjuvant chemotherapy (NCT); Time to surgery; Prognosis

Submitted Apr 11, 2019. Accepted for publication Aug 08, 2019.

doi: $10.21037 /$ tcr.2019.08.42

View this article at: http://dx.doi.org/10.21037/tcr.2019.08.42

\section{Introduction}

Neoadjuvant chemotherapy (NCT) followed by curative resection has been increasingly adopted to prolong the survival of patients with locally advanced gastric cancer (GC) through the ability of NCT to reduce micrometastases, downstage the tumor, and improve tumor resectability (1-4). In the era of multimodal therapy, surgeons are frequently confronted with the challenge of scheduling surgery at an appropriate time after the completion of NCT. Patients with incomplete resolution of chemotherapy-related 
toxicity, a worsened nutrition status or serious comorbidities are more likely to fare poorly after premature surgery $(5,6)$. However, concomitant with surgery postponement is potentially impaired prognosis, psychological distress, and a worsened quality of life $(7,8)$. In clinical practice, primary tumor resection is usually scheduled within a few weeks from the last dose of preoperative chemotherapy, but the optimal time interval between the end of NCT and definitive surgery has not yet been defined.

The time interval from the completion of NCT to definitive surgery is arbitrarily decided in most trials that have investigated the survival benefit of NCT $(1,9)$. To the best of our knowledge, only one retrospective study has examined gastrectomy timing after NCT, suggesting that a longer NCT-surgery interval increased the odds of a pathologically complete response (pCR) but had no influence on survival (10).

Whereas preclinical models have confirmed biologically better outcomes with shorter intervals between surgical treatment and the initiation of adjuvant chemotherapy (11-13), no such biological evidence was in favor of a shorter interval between the completion of NCT and surgery. We hypothesized that a longer interval would not impair survival based on the ability of NCT to systemically eliminate micrometastases before surgery.

\section{Methods}

\section{Study population}

Patients with locally advanced GC (T3/T4, or N+) who underwent NCT followed by gastrectomy between 2006 and 2016 at a high-volume tertiary referral center were retrospectively reviewed. The exclusion criteria were as follows: (I) active synchronous tumors; (II) urgent symptoms; (III) received NCT outside of our institution; (IV) underwent radiotherapy in addition to chemotherapy; and (V) unevaluable TTS. Urgent symptoms referred to symptoms that warranted interventions, including bleeding, ileus, and gastric pyloric obstruction. The Institutional Review Board of the National Cancer Center/Cancer Hospital has reviewed and approved this study and has also agreed that individual patient consent was not required to report clinical outcomes alone. The outcomes of this study did not affect the management of the patients. The patients' personal data have been secured.

\section{Chemotherapy and surgical treatment}

Preoperative chemotherapy according to the SOX (S-1 $80 \mathrm{mg} / \mathrm{m}^{2}$ on days $1-14$ and oxaliplatin $130 \mathrm{mg} / \mathrm{m}^{2}$ on day 1 ) or XELOX regimen (capecitabine $1,000 \mathrm{mg} / \mathrm{m}^{2}$ on days $1-14$ and oxaliplatin $130 \mathrm{mg} / \mathrm{m}^{2}$ on day 1) was performed with four 3-week cycles. Alternatively, paclitaxel $\left(50 \mathrm{mg} / \mathrm{m}^{2}\right)$ was added to the SOX or XELOX regimen for patients with a good physical condition. Dosage reduction or withdrawal was applied in the case of severe adverse events during chemotherapy according to the clinician's decision. The antitumor effect was assessed according to Response Evaluation Criteria in Solid Tumors 1.1 (RECIST 1.1) every two cycles (14). When tumor reduction was detected, the NCT was continued for another two cycles. Otherwise, gastrectomy or switching to other regimen was considered after obtaining informed consent and approval from patients. Surgery was usually performed within 5-6 weeks after the last dose of preoperative chemotherapy. Subtotal or total gastrectomy plus D2 lymphadenectomy was performed as required by the guidelines of the Japanese Gastric Cancer Association (15). Additional organ resection was indicated in the case of tumor involvement with adjacent organs, including resection of the spleen, colon, pancreas and liver, in addition to gastrectomy. Adjuvant chemotherapy was initiated 4 weeks after surgery using the same protocol as preoperatively. The adjuvant component was cancelled or postponed in the case of severe chemotherapy toxicity, postoperative complication, a worsened nutrition status, or other reasons.

\section{Evaluation of clinicopathological variables}

Patients were divided into three groups according to TTS: (I) $\leq 4$ weeks; (II) 5-6 weeks; and (III) $>6$ weeks. The patient and clinicopathological characteristics, including age, gender, comorbidities, American Society of Anesthesiologists (ASA) risk score, clinical stage, NCT regimen and cycles, severe NCT toxicity, extent of gastrectomy, additional organ resection, operative duration, intraoperative blood loss, postoperative complications, length of postoperative hospital stay, examined lymph nodes, resection margin, differentiation, $\mathrm{pT}, \mathrm{pN}$, pStage, Mandard score, adjuvant chemotherapy and cycles were compared between the groups. 


\section{Postoperative therapy and follow up}

After discharge, the patients were required to visit either the clinics of the medical oncologist at our center or that of a resident oncologist for postoperative therapy and follow up. All the patients were required to visit the clinics every 3 months during the first 2 postoperative years, every 6 months thereafter for 3 years, and yearly after 5 years. Recurrence and death were determined from hospital records or from telephone interviews.

\section{Statistical analysis}

To reduce the bias in the pretreatment waiting time, progression-free survival (PFS) and overall survival (OS) were defined as the time interval from the start of preoperative chemotherapy, rather than the date of diagnosis, to the date of the first documented recurrence or death, respectively. Categorical variables were analyzed using chi-squared or Fisher's exact test, and continuous data were analyzed using Student's $t$ test or the Mann-Whitney U test. Survival was assessed by Kaplan-Meier estimates and was compared using the log-rank test. Cox regression models were applied to explore the association between TTS and survival outcomes after adjustment for potential confounders. All statistical tests were conducted using SPSS version 22.0 (SPSS, Inc., Chicago, IL, USA). Statistical significance was set at 2 -sided $\mathrm{P}<0.05$.

\section{Results}

The patient and clinical characteristics are summarized in Table 1. Seventy of two hundred twenty-nine patients $(30.6 \%)$ had surgery within 4 weeks after their last dose of NCT, 103 (45.0\%) within 5-6 weeks, and 56 (24.5\%) after 6 weeks. The median age was 56.0 [interquartile range (IQR), 47.0-63.0] years, and the median TTS was 34.0 (IQR 26.0-42.0) days. One hundred twenty-eight patients (55.9\%) received SOX/XELOX chemotherapy, and 101 $(44.1 \%)$ received paclitaxel in addition to SOX/XELOX. One hundred thirty-one $(57.2 \%)$ patients completed all the intended cycles of preoperative chemotherapy, 165 (72.0\%) received adjuvant chemotherapy, and 86 of 165 (52.1\%) completed all the intended cycles of postoperative chemotherapy. We analyzed the reasons for surgery delay among patients with TTS $>6$ weeks (Table 2). They were chemotherapy-related toxicity, severe postoperative complication, poor performance status and nutritional support requirement. Other reasons included patients' choice, economic reasons, and logistic reasons.

The three groups did not significantly differ in terms of age, gender, comorbidities, ASA risk score, clinical stage, tumor location, clinical response and treatment-related toxicity except for the NCT regimen $(\mathrm{P}=0.010)$ and number of cycles of NCT $(\mathrm{P}=0.017)$. Multivariate analysis showed that the NCT regimen was the only independent factor associated with TTS $>6$ weeks (Table 3). The 3 groups did not differ significantly regarding most surgical and histopathological characteristics except for the pathological stage $(\mathrm{P}=0.015)$ (Table 4).

The median follow-up time for the 229 patients was 28.3 (IQR, 15.7-51.4) months. The median PFS was 31.0 [95\% confidence interval (CI), 16.3-45.6] months, and the median OS was 68.6 (95\% CI, not reached) months. The median time to death after the diagnosis of recurrence was 7.0 (IQR, 2.3-14.3) months. For the whole cohort, the 3 -year PFS and OS rates were $49.9 \%$ and $61.5 \%$, respectively. The 3-year PFS estimates were $41.9 \%, 42.8 \%$ and $61.7 \%$ in patients who underwent surgery in $\leq 4,5-6$, and $>6$ weeks after NCT, respectively $(\mathrm{P}=0.044)$. The 3 -year OS estimates were $57.7 \%, 58.0 \%$ and $68.2 \%$ in the three groups, respectively $(\mathrm{P}=0.202)$. Figure $1 A, B$ demonstrated Kaplan-Meier estimates of PFS and OS according to TTS.

In multivariable analysis, the extent of gastrectomy (total), tumor differentiation (poor/undifferentiated), pathological $(\mathrm{T})$ stage $(\mathrm{T} 3 / \mathrm{T} 4), \mathrm{pN}$ stage $(\mathrm{N}+)$, resection margin (R1), and adjuvant therapy (none/incomplete) were independently associated with poor OS (Table 5). The extent of gastrectomy (total), tumor differentiation (poor/ undifferentiated), $\mathrm{pN}$ stage $(\mathrm{N}+)$, and resection margin (R1) were independently associated with poor PFS (Table 6).

\section{Discussion}

The present study found that the NCT regimen is the only independent factor associated with prolonged TTS ( $>6$ weeks). TTS had no impact on the histopathological response or survival outcomes of patients with locally advanced GC who underwent preoperative chemotherapy.

The time interval to surgery is an important question frequently asked by patients but is also a question without a definite conclusion. Seminal trials whose results led to the introduction of preoperative chemotherapy into GC usually scheduled surgery within 4 and 6 weeks after the last dose of chemotherapy $(1,3,9)$. However, no clinical trial has defined 
Table 1 Patient and clinical characteristics by the interval from the completion of neoadjuvant therapy to surgery

\begin{tabular}{|c|c|c|c|c|c|}
\hline Characteristic & $\begin{array}{l}\text { Total patients } \\
\quad(\mathrm{n}=229)\end{array}$ & $\begin{array}{l}\text { Time to surgery } \\
\leq 4 \text { weeks }(n=70)\end{array}$ & $\begin{array}{l}\text { Time to surgery } \\
5-6 \text { weeks }(n=103)\end{array}$ & $\begin{array}{l}\text { Time to surgery } \\
>6 \text { weeks }(n=56)\end{array}$ & $P$ value \\
\hline Time to surgery (day), median (IQR) & $34.0(26.0-42.0)$ & $23.5(18.8-25.0)$ & $35.0(31.0-39.0)$ & $53.5(46.3-60.8)$ & $<0.001$ \\
\hline Age (years), median (IQR) & $56.0(47.0-63.0)$ & $58.0(48.8-64.0)$ & $55.0(44.0-62.0)$ & $57.0(50.0-64.0)$ & 0.196 \\
\hline Gender & & & & & 0.188 \\
\hline Comorbidity & & & & & 0.436 \\
\hline No & 158 & 48 & 70 & 40 & \\
\hline 1 & 53 & 14 & 28 & 11 & \\
\hline $3-4$ & 22 & 8 & 11 & 3 & \\
\hline Clinical stage & & & & & 0.133 \\
\hline IIIA & 44 & 10 & 22 & 12 & \\
\hline IIIB & 95 & 26 & 43 & 26 & \\
\hline IIIC & 90 & 34 & 38 & 18 & \\
\hline NCT regimen & & & & & 0.010 \\
\hline SOX/XELOX & 128 & 42 & 47 & 39 & \\
\hline Middle & 62 & 19 & 27 & 16 & \\
\hline Lower & 113 & 32 & 52 & 29 & \\
\hline Clinical response $^{a}$ & & & & & 0.757 \\
\hline PR & 158 & 50 & 69 & 39 & \\
\hline SD/PD & 71 & 20 & 34 & 17 & \\
\hline Severe NCT toxicity ${ }^{\mathrm{b}}$ & 87 & 25 & 37 & 25 & 0.498 \\
\hline
\end{tabular}

${ }^{a}$, according to the Response Evaluation Criteria in Solid Tumors 1.1 (RECIST 1.1); ${ }^{\text {, }}$, according to the National Cancer Institute Common Terminology Criteria for Adverse Events (version 4.0). IQR, interquartile range; ASA, American Society of Anesthesiologists; NCT, neoadjuvant chemotherapy; SOX, oxaliplatin + S-1; XELOX, oxaliplatin + capecitabine; PR, partial remission; PD, progressive disease; SD, stable disease.

the optimal timing of surgery after NCT and the current clinical guidelines do not present specific guidelines on an optimal interval. In default of relevant data, clinicians had to extrapolate from interval data in the context of adjuvant therapy, although its applicability in preoperative settings had not been validated (16-19). 
Table 2 Reasons for surgery delay among patients with time to surgery (TTS) $>6$ weeks

\begin{tabular}{lc}
\hline Reasons & Number of patients (\%) \\
\hline Hematologic toxicity & $15(26.8)$ \\
Gastrointestinal symptoms & $12(21.4)$ \\
Nutritional support requirement & $7(12.5)$ \\
Poor performance status & $9(16.1)$ \\
Patient choice & $4(7.1)$ \\
Logistic reasons & $4(7.1)$ \\
Economic reasons & $2(3.6)$ \\
Unknown & $3(5.4)$ \\
\hline
\end{tabular}

In contrast to previous results from esophageal, rectal and GC showing that prolonged TTS significantly increased the odds of pCR (10,20-22), we did not observe any impact of TTS on the tumor histopathological response or tumor downstaging. Data on the influence of delaying TTS on the pathologic response rate are conflicting. An increased time interval ( $>6$ weeks) between NCT and surgery revealed a significantly increased pCR rate among patients with locally advanced GC in a study conducted by Liu and colleagues (10). They speculated that a prolonged NCT-surgery interval during which tissue swelling and local inflammation induced by chemotherapy was allowed to subside could lead to a greater extent of tumor regression (e.g., downsizing and downstaging) through apoptosis and necrosis. In the current study, the association between the TTS and pathological response was not observed. The rate of pCR was $22.7 \%$ in the study conducted by Liu and colleagues compared with $7.0 \%$ in our study, although more than $40 \%$ of patients were given paclitaxel in addition to platinum plus fluorouracil chemotherapy. The reason for such an uncommon high probability of pCR in the study by Liu et al. is unclear. Investigating whether prolonged TTS increased the odds of pCR was not allowed due to the inferior chemosensitivity of GC and limited cases of complete responders in the current single institutional cohort. A multi-institutional study is needed to address this issue in the future.

There was a concern that delayed surgical treatment after NCT can lead to a poor survival outcome. The survival outcomes in our study did not differ across groups, a finding that was supported by previous studies, suggesting that postponing surgery beyond the currently accepted schedule
Table 3 Independent factors associated with TTS $>6$ weeks

\begin{tabular}{lcc}
\hline Variables & OR $(95 \% \mathrm{Cl})$ & $\mathrm{P}$ value \\
\hline Number of NCT cycles & $1.071(0.876-1.308)$ & 0.504 \\
NCT regimen & $0.444(0.230-0.854)$ & 0.015 \\
\hline TTS, time to surgery; NCT, neoadjuvant chemotherapy; OR, \\
odds ratio.
\end{tabular}

did not impair survival. The association between TTS and survival varied with the cancer type. TTS had been shown to impair survival in many cancers, such as breast, esophageal, rectal and ovarian cancers. Sanford et al. analyzed data from a large breast cancer patient cohort treated with NCT at a single institution, revealing worse outcomes when surgery was scheduled after longer than 8 weeks (23). A recent study correlated the time interval between the end of NCT and initiation of adjuvant chemotherapy to survival in patients with stage III or IV ovarian cancer. The longer time intervals were independently predictive of higher risks of recurrence and death (24). In esophageal cancer, the perioperative mortality and OS are significantly correlated with the time interval between preoperative chemoradiation therapy and esophagectomy (25). Therefore, the correlation between TTS and prognosis vary with the cancer type, and this was probably due to distinct tumor characteristics and treatments.

The prolonged interval between NCT and surgery is driven by multiple factors. One of the most frequently encountered is the time period required for the resolution of short-term chemotherapy toxicities. Chemotherapy-related toxicities is a well-known contributor to postoperative complications after immediate surgery (5). Poor physical conditions and comorbidities of patients may also prolong this time interval, explaining why a difference was not detected in TTS between patients with or without NCT toxicity. Patients would experience psychological pressure, a deteriorated quality of life and satisfaction worrying about an impaired survival during the delay $(7,8)$. No negative influence of postponed surgery on postoperative complications or survival suggested by our results should reassure patients and surgeons when scheduling surgery after NCT, but it did not support delaying surgery without reason. If a prolonged preoperative wait time is necessary for recovery from chemotherapy-related toxicity, physical condition improvement or comorbidity control, active surveillance is warranted and the initiation of treatment should occur as soon as possible. 
Table 4 Surgical and histopathological characteristics by the interval from the completion of neoadjuvant chemotherapy to surgery.

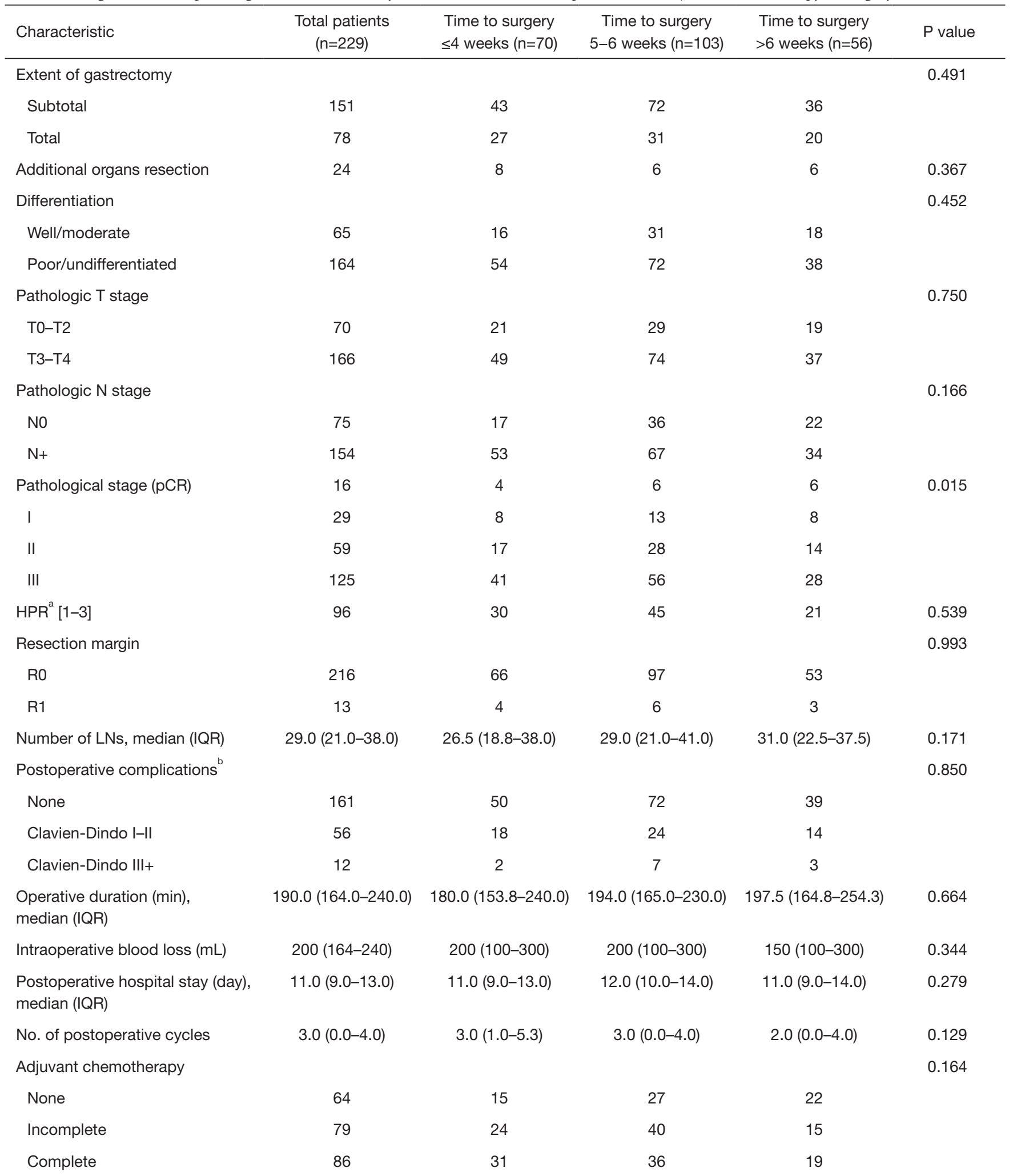

a , according to Mandard score; ${ }^{b}$, according to the Clavien-Dindo classification system. pCR, pathologically complete response; HPR, histopathological response; LNs, lymph nodes; IQR, interquartile range. 


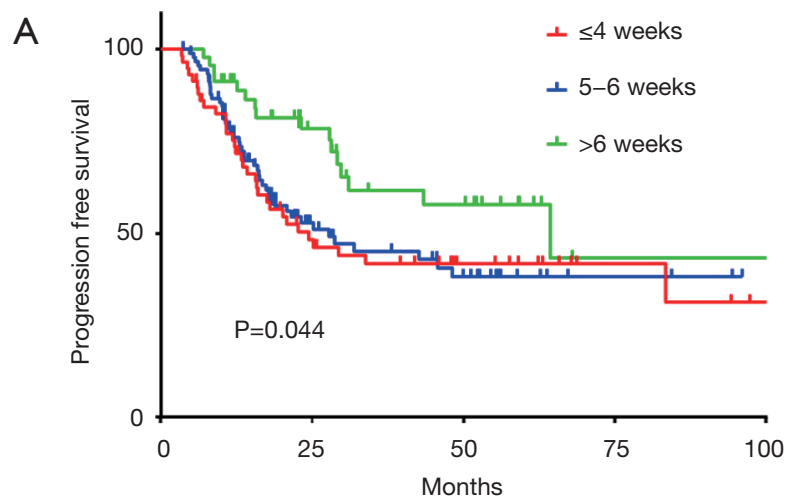

$\begin{array}{lccccc}\leq 4 \text { weeks } & 70 & 28 & 17 & 6 & 3 \\ \text { 5-6 weeks } & 103 & 34 & 18 & 6 & 3 \\ >6 \text { weeks } & 56 & 32 & 19 & 6 & 5\end{array}$

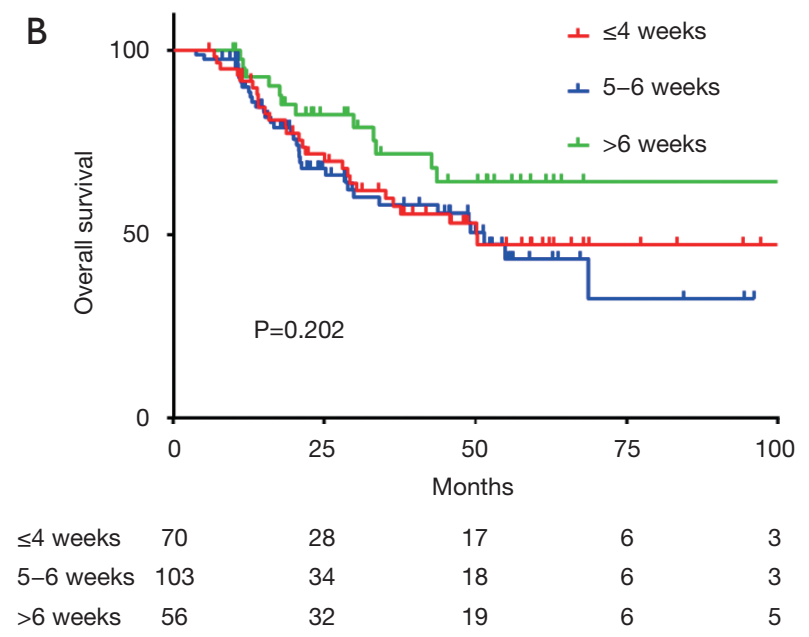

Figure 1 Kaplan-Meier analyses of progression-free survival (A) and overall survival (B) according to time to surgery.

Table 5 Univariate and multivariate analysis of the factors affecting OS

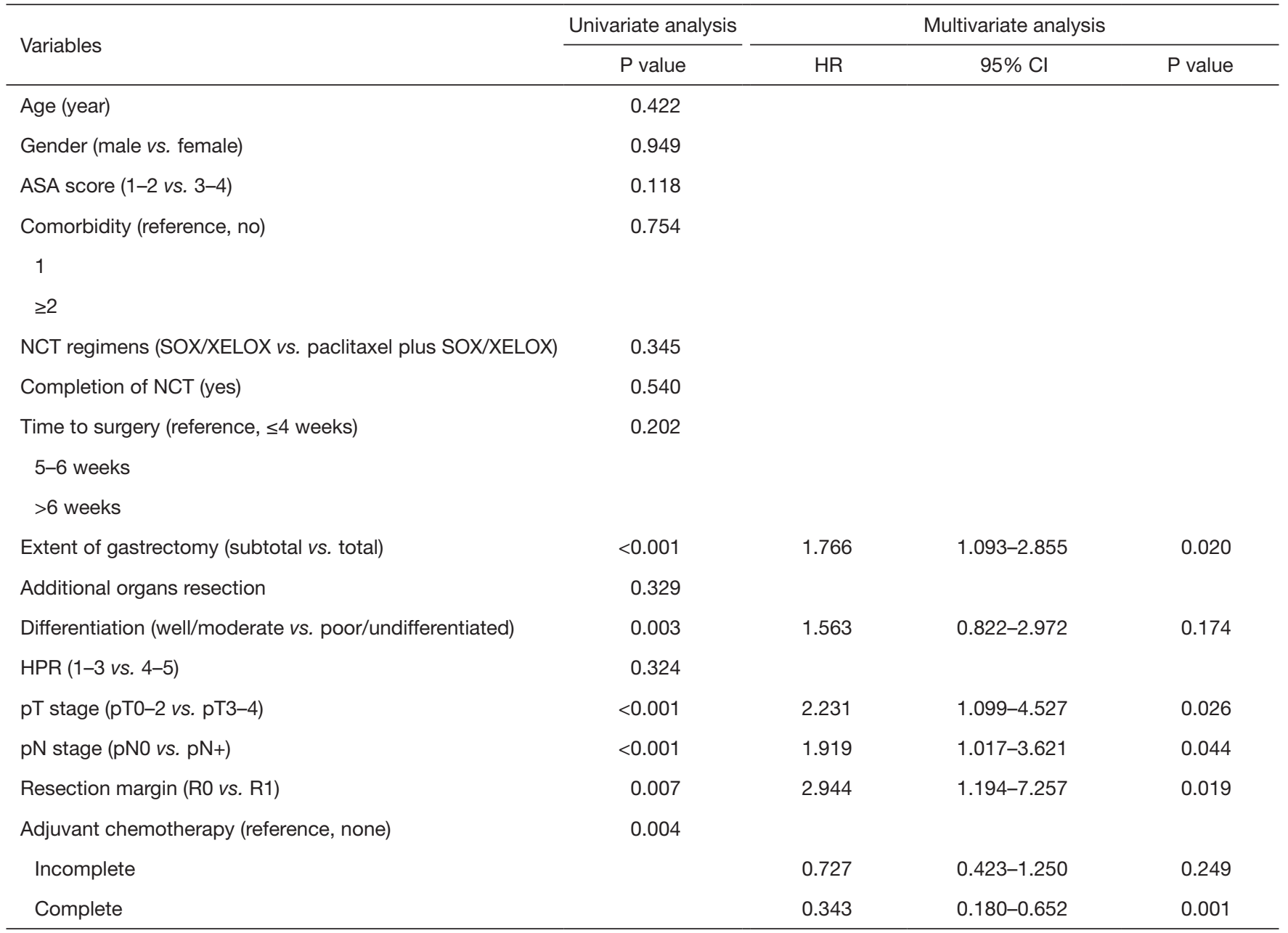

OS, overall survival; HR, hazards ratio; Cl, confidence interval; ASA, American Society of Anesthesiologists; NCT, neoadjuvant chemotherapy; SOX, oxaliplatin + S-1; XELOX, oxaliplatin + capecitabine; HPR, histopathological response. 
Table 6 Univariate and multivariate analysis of the factors affecting PFS

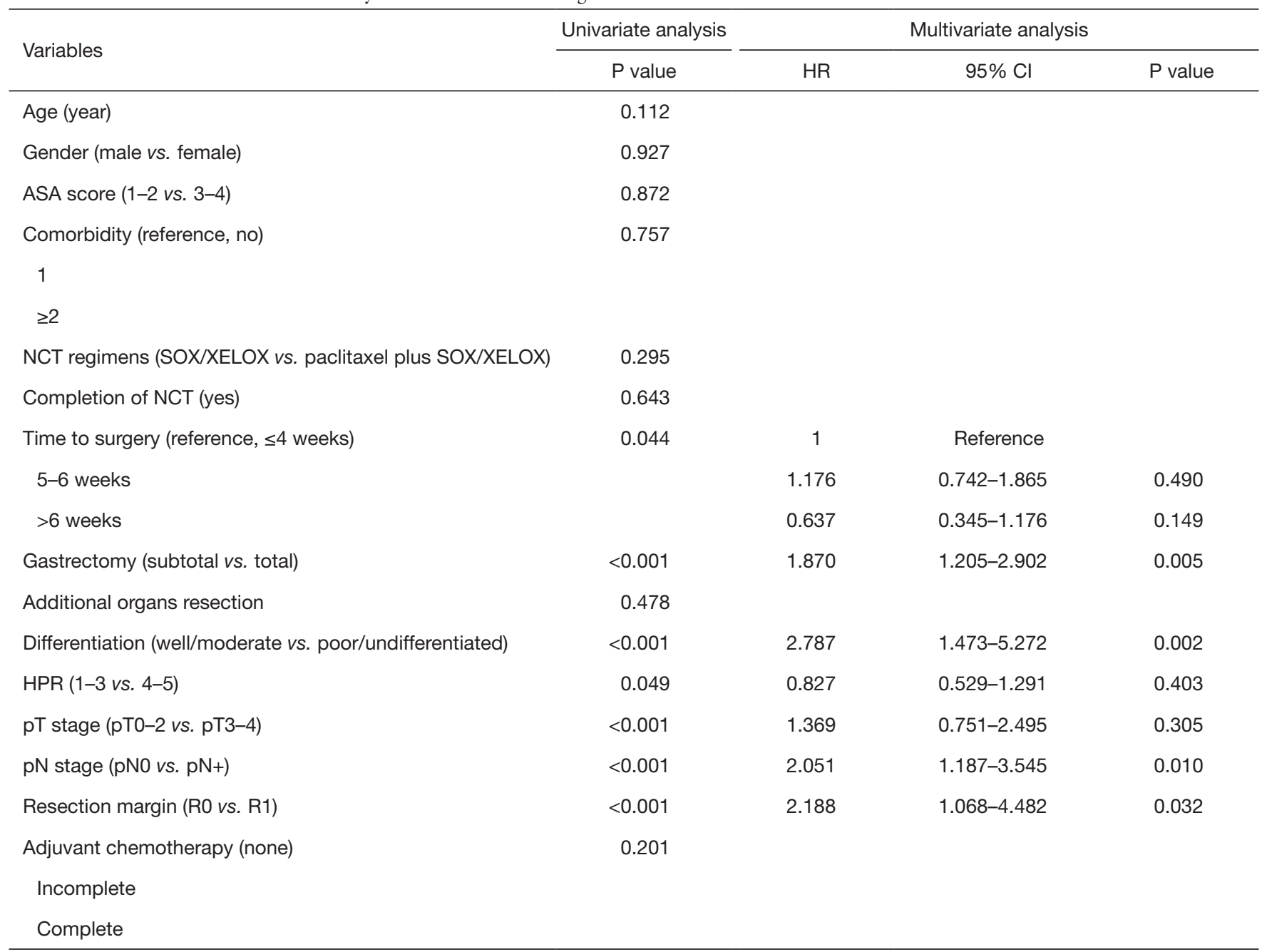

PFS, progression-free survival; HR, hazards ratio; Cl, confidence interval; ASA, American Society of Anesthesiologists; NCT, neoadjuvant chemotherapy; SOX, oxaliplatin + S-1; XELOX, oxaliplatin + capecitabine; HPR, histopathological response.

There are several inherent limitations that should be noted. Patients who underwent neoadjuvant radiotherapy were excluded because they were inherently different from those receiving chemotherapy alone. Resulting from the paucity of high-level evidence regarding a survival benefit for NCT plus surgery over surgery followed by adjuvant chemotherapy in patients with locally advanced GC, patients treated with NCT at our center mostly had clinically stage III disease as they carry a high risk of poor prognosis. This precluded our conclusion from being solid enough among patients with less advanced disease. The ethnicity, chemotherapy regimen, and postoperative treatment modality in the current study were also different from those in European studies. The range of TTS was narrow, and the selection of cut-off points were arbitrary to some extent. Patients could differ by as little as two weeks between the first and third tertiles because patients undergoing radiotherapy whose preoperative waiting time was usually longer were excluded because they were inherently different. The arbitrary cut-off points were selected based on previous retrospective studies addressing the same issue demonstrating that TTS $>6$ weeks increased the probability of pCR. TTS is usually $5-6$ weeks, which is most commonly adopted by clinicians in China. Although the longest TTS group was the least likely to receive paclitaxel, the survival curves seem to be better than those of the other two groups together in the univariate analysis. However, TTS was not recognized as a significantly 
independent predictor of survival in the multivariate analysis. We believed that the limited number of patients with the longest TTS ( $>6$ weeks), a narrow range of TTS and relatively short follow-up time in this study might prevent us from reaching a statistically significant result. A large-sampled multi-institutional cohort is warranted to further investigate this issue. Finally, the median followup period was relatively short. However, because the first recurrence of GC usually develops within the first two years postoperatively, the follow-up period may have been sufficient to draw a conclusion (26).

\section{Conclusions}

Our findings suggest that TTS did not impact the histopathological response or survival outcomes. It reassures surgeons to delay surgery for patients experiencing chemotherapy-toxicity, a worsened nutritional status or serious comorbidities. On the other hand, when the patient is fit for surgery, delaying surgery appears to bear no additional benefits. Conclusive evidence from a clinical trial are necessary to define the optimal timing of surgery after NCT and to determine whether prolonged TTS could benefit patients.

\section{Acknowledgments}

Funding: This study was supported by a grant from National Key R\&D Program of China (Grant No. 2017YFC0908300).

\section{Footnote}

Conflicts of Interest: All authors have completed the ICMJE uniform disclosure form (available at http://dx.doi. org/10.21037/tcr.2019.08.42). The authors have no conflicts of interests to declare.

Ethical Statement: The authors are accountable for all aspects of the work in ensuring that questions related to the accuracy or integrity of any part of the work are appropriately investigated and resolved. This study was conducted in accordance with the Declaration of Helsinki (as revised in 2013). The Institutional Review Board of National Cancer Center/ National Clinical Research Center for Cancer/Cancer Hospital has reviewed and approved this study, and has also agreed that individual patient consent was not required to report clinical outcomes alone (No. 17-156/1412).

Open Access Statement: This is an Open Access article distributed in accordance with the Creative Commons Attribution-NonCommercial-NoDerivs 4.0 International License (CC BY-NC-ND 4.0), which permits the noncommercial replication and distribution of the article with the strict proviso that no changes or edits are made and the original work is properly cited (including links to both the formal publication through the relevant DOI and the license). See: https://creativecommons.org/licenses/by-nc-nd/4.0/.

\section{References}

1. Cunningham D, Allum WH, Stenning SP, et al. Perioperative chemotherapy versus surgery alone for resectable gastroesophageal cancer. $\mathrm{N}$ Engl J Med 2006;355:11-20.

2. Ajani JA, Mansfield PF, Janjan N, et al. Multi-institutional trial of preoperative chemoradiotherapy in patients with potentially resectable gastric carcinoma. J Clin Oncol 2004;22:2774-80.

3. Schuhmacher C, Gretschel S, Lordick F, et al. Neoadjuvant chemotherapy compared with surgery alone for locally advanced cancer of the stomach and cardia: European Organisation for Research and Treatment of Cancer randomized trial 40954. J Clin Oncol 2010;28:5210-8.

4. Oki E, Emi Y, Kusumoto T, et al. Phase II study of docetaxel and S-1 (DS) as neoadjuvant chemotherapy for clinical stage III resectable gastric cancer. Ann Surg Oncol 2014;21:2340-6.

5. Robb WB, Messager M, Gronnier C, et al. HighGrade Toxicity to Neoadjuvant Treatment for Upper Gastrointestinal Carcinomas: What is the Impact on Perioperative and Oncologic Outcomes? Ann Surg Oncol 2015;22:3632-9.

6. Charalampakis N, Xiao L, Lin Q, et al. Co-morbidities Rather than Age Impact Outcomes in Patients Receiving Preoperative Therapy for Gastroesophageal Adenocarcinoma. Ann Surg Oncol 2017;24:2291-301.

7. Gray RE, Fitch MI, Phillips C, et al. Presurgery experiences of prostate cancer patients and their spouses. Cancer Pract 1999;7:130-5.

8. Robinson KM, Christensen KB, Ottesen B, et al. Diagnostic delay, quality of life and patient satisfaction among women diagnosed with endometrial or ovarian 
cancer: a nationwide Danish study. Qual Life Res 2012;21:1519-25.

9. Schuhmacher C, Gretschel S, Lordick F, et al. Neoadjuvant Chemotherapy Compared With Surgery Alone for Locally Advanced Cancer of the Stomach and Cardia: European Organisation for Research and Treatment of Cancer Randomized Trial 40954. J Clin Oncol 2010;28:5210-8.

10. Liu Y, Zhang KC, Huang XH, et al. Timing of surgery after neoadjuvant chemotherapy for gastric cancer: Impact on outcomes. World J Gastroenterol 2018;24:257-65.

11. Gunduz N, Fisher B, Saffer EA. Effect of surgical removal on the growth and kinetics of residual tumor. Cancer Res 1979;39:3861-5.

12. Fisher B, Gunduz N, Coyle J, et al. Presence of a growthstimulating factor in serum following primary tumor removal in mice. Cancer Res 1989;49:1996-2001.

13. Segatto I, Berton S, Sonego M, et al. Surgery-induced wound response promotes stem-like and tumor-initiating features of breast cancer cells, via STAT3 signaling. Oncotarget 2014;5:6267-79.

14. Watanabe H, Okada M, Kaji Y, et al. New response evaluation criteria in solid tumours-revised RECIST guideline (version 1.1). Gan To Kagaku Ryoho 2009;36:2495-501.

15. Japanese Gastric Cancer Association. Japanese gastric cancer treatment guidelines 2014 (ver. 4). Gastric Cancer 2017;20:1-19.

16. Fujitani K, Kurokawa Y, Takeno A, et al. Time to initiation or duration of S-1 adjuvant chemotherapy; which really impacts on survival in stage II and III gastric cancer? Gastric Cancer 2018;21:446-52.

17. Yamamoto M, Sakaguchi Y, Kinjo N, et al. S-1 Adjuvant Chemotherapy Earlier After Surgery Clinically Correlates with Prognostic Factors for Advanced Gastric Cancer. Ann Surg Oncol 2016;23:546-51.

Cite this article as: $\mathrm{Wu} \mathrm{C}$, Zhou $\mathrm{H}$, Wang T, Zhang $\mathrm{X}$, Chen Y, Zhao D. Impact of the time from the completion of neoadjuvant chemotherapy to surgery on the outcomes of patients with gastric cancer. Transl Cancer Res 2019;8(5):18531862. doi: $10.21037 /$ tcr.2019.08.42
18. Greenleaf EK, Kulaylat AN, Hollenbeak CS, et al. Timing of Adjuvant Chemotherapy and Impact on Survival for Resected Gastric Cancer. Ann Surg Oncol 2016;23:4203-13.

19. Sakuramoto S, Sasako M, Yamaguchi T, et al. Adjuvant chemotherapy for gastric cancer with $\mathrm{S}-1$, an oral fluoropyrimidine. N Engl J Med 2007;357:1810-20.

20. Shapiro J, van Hagen P, Lingsma HF, et al. Prolonged time to surgery after neoadjuvant chemoradiotherapy increases histopathological response without affecting survival in patients with esophageal or junctional cancer. Ann Surg 2014;260:807-13.

21. Macchia G, Gambacorta MA, Masciocchi C, et al. Time to surgery and pathologic complete response after neoadjuvant chemoradiation in rectal cancer: A population study on 2094 patients. Clin Transl Radiat Oncol 2017;4:8-14.

22. van der Werf LR, Dikken JL, van der Willik EM, et al. Time interval between neoadjuvant chemoradiotherapy and surgery for oesophageal or junctional cancer: A nationwide study. Eur J Cancer 2018;91:76-85.

23. Sanford RA, Lei X, Barcenas CH, et al. Impact of Time from Completion of Neoadjuvant Chemotherapy to Surgery on Survival Outcomes in Breast Cancer Patients. Ann Surg Oncol 2016;23:1515-21.

24. Lee YJ, Chung YS, Lee JY, et al. Impact of the time interval from completion of neoadjuvant chemotherapy to initiation of postoperative adjuvant chemotherapy on the survival of patients with advanced ovarian cancer. Gynecol Oncol 2018;148:62-7.

25. Franko J, Voynov G, Goldman CD. Esophagectomy Timing After Neoadjuvant Therapy for Distal Esophageal Adenocarcinoma. Ann Thorac Surg 2016;101:1123-30.

26. Baiocchi GL, Marrelli D, Verlato G, et al. Follow-up after gastrectomy for cancer: an appraisal of the Italian research group for gastric cancer. Ann Surg Oncol 2014;21:2005-11. 